\title{
Unaware yet reliant on attention: Experience sampling reveals that mind-wandering impedes implicit learning
}

\author{
Michael S. Franklin $^{1}$ • Jonathan Smallwood ${ }^{2}$ - Claire M. Zedelius ${ }^{1}$ • \\ James M. Broadway ${ }^{1}$ • Jonathan W. Schooler ${ }^{1}$
}

Published online: 30 June 2015

(C) Psychonomic Society, Inc. 2015

\begin{abstract}
Although implicit learning has been widely studied, controversy remains regarding its reliance on attentional resources. A central issue in this controversy is the question of how best to manipulate attention. The usual approach of comparing implicit learning in a serial reaction time (SRT) task under single- versus dual-task conditions is known to be problematic, because the secondary task may not only divert attention away from the primary task, but also interfere with the implicit-learning process itself. To address this confound, in the present study we used an experience-sampling instead of a dual-task approach. We assessed lapses of attention (mindwandering) with experience-sampling thought probes during a standard implicit-learning SRT task. The results revealed a significant negative correlation between mind-wandering and implicit learning. Thus, greater task focus was associated with improved implicit sequence learning. This result suggests that, at least in the context of this SRT task, optimal implicit learning relies on attention.
\end{abstract}

Keywords Implicit learning · Mind-wandering $\cdot$ Control processes - Automatic processing - Conscious awareness . Sequence learning $\cdot$ Serial reaction time

When conceptualizing the learning process, researchers often distinguish between explicit learning, in which a person can describe the learned material, and implicit learning, in which a

Michael S. Franklin

franklin@psych.ucsb.edu

1 Department of Psychological and Brain Sciences, University of California, Bldg 551, Room 1304, Santa Barbara, CA 93106-9660, USA

2 Department of Psychology, University of York, York, UK person, although unaware of what has been learned, still shows performance benefits. The dissociation between these two types of learning is most clearly demonstrated in patients suffering damage to the medial temporal lobe. This damage can result in anterograde amnesia, in which explicit learning is compromised, yet implicit learning is spared (i.e., a new skill can be acquired, in spite of memory loss of even the learning session itself). Although a large number of studies of implicit learning have been informative regarding its cognitive and neural mechanisms (e.g., Keele, Ivry, Mayr, Hazeltine, \& Heuer, 2003; Nissen \& Bullemer, 1987; Seger, 1994), there is still a lack of consensus regarding the role of attentional resources in such learning. Although some studies have suggested that implicit sequence learning can proceed with little or no use of attentional resources (Cleeremans \& Jiménez, 1998; Frensch, Lin, \& Buchner, 1998), other studies have shown impaired implicit learning when attention is diverted away from the primary implicit-learning task (Shanks \& Channon, 2002; Shanks, Rowland, \& Ranger, 2005). Still other results support the view that inattention may actually improve implicit learning (Filoteo, Lauritzen, \& Maddox, 2010; Nemeth, Janacsek, Polner, \& Kovacs, 2013; though see Newell, Moore, Willis, \& Milton, 2013, for a reevaluation of Filoteo et al., 2010).

The question of whether implicit learning requires attentional resources has typically been addressed through the use of a serial reaction time (SRT) task (Nissen \& Bullemer, 1987). In this task, participants identify the location of a target and press a corresponding button depending on the location of the target. For example, if an asterisk appears in the first of four squares arranged from left to right, the participant should press the far left buttonpress (Nissen \& Bullemer, 1987). A key aspect of the SRT task is that, although some target locations are determined randomly, fixed patterns are embedded into the sequential presentation of targets, and the patterns 
repeat over the course of the task. By comparing the RTs from patterned versus random sequences, it is possible to reveal that implicit learning has taken place. That is, it is usually found that, toward the end of the task, participants are faster when responding to patterned than to random sequences. Importantly, despite this RT advantage, participants are unable to reconstruct the pattern and usually report no awareness of it, ruling out explicit learning.

To investigate the role of attention in implicit learning, researchers typically compare implicit learning under singleversus dual-task conditions, with the assumption being that when a "distractor" task is performed, attention is relatively more often diverted away from the primary task. Although the findings have been mixed, much of the early work using this approach has come under question. A main argument is that the secondary task may not only divert attention away from the task, but also interfere with the learning process itself (e.g., Frensch et al., 1998; Stadler, 1995). For instance, by presenting distractor stimuli in the SRT task, or by introducing additional operations to be performed on the stimuli of the SRT task, the interval between stimuli in the SRT task is filled, which it has been suggested limits the simultaneous availability of sequence elements in working memory (Schmidtke \& Heuer, 1997). Although recent work has modified the protocol, even the improved methodology does not completely rule out this option (see Shanks et al., 2005). Even on a theoretical level, it remains unclear whether it is possible to design the kind of distractor task that is difficult enough to sufficiently tax the attentional resources required for learning, yet that does not put significant constraints on performing the SRT task in the same manner as it is performed under single-task conditions.

In the present study, we took a novel approach to investigate the impact of attention on implicit learning. Specifically, we used experience sampling during the SRT task to assess the degree to which attention was focused on the task. The idea behind this methodology is derived from the literature on mind-wandering, which is concerned with the occurrence of task-unrelated thoughts under single-task conditions (Teasdale et al., 1995; Teasdale, Proctor, Lloyd, \& Baddeley, 1993). Studies have shown that people's attention becomes disengaged from the task and shifts to task-unrelated personal concerns $30 \%-50 \%$ of the time (Kane et al., 2007; Killingsworth \& Gilbert, 2010). The task-unrelated thoughts measured by thought probes are usually associated with measurable performance deficits across a variety of different tasks (see Mooneyham \& Schooler, 2013; Smallwood \& AndrewsHanna, 2013, for reviews), such as reading (Franklin, Smallwood, \& Schooler, 2011; Schooler, Reichle, \& Halpern, 2004; Smallwood, McSpadden, \& Schooler, 2008), simple motor tasks (Seli et al., 2014), and tasks requiring sustained attention and executive processing (e.g., the sustained-attention-to-response task-Smallwood et al.,
2008; or Stroop task-Thomson, Besner, \& Smilek, 2013), as well as learning and memory tasks (Mrazek et al., 2012; Smallwood, Obonsawin, \& Heim, 2003). On the basis of this literature, we expected that, if implicit learning depends on attention, the thought probe approach should be helpful to reveal it.

In a way, responding to thought probes embedded into the SRT task created a dual-task context. However, since all participants responded to thought probes, any potential negative (or positive) effect of this secondary task on the implicitlearning process should not be specific to the distracted participants.

The impact of mind-wandering on implicit learning is, as of yet, an open question, with each of three outcomes - interference, no effect, or facilitation - being theoretically plausible. Given the many previously documented examples of performance deficits associated with mind-wandering (especially with regard to learning and memory tasks), one might suspect that it would also impair implicit learning. However, since incidental or implicit learning occurs outside of conscious awareness, it has been suggested that such learning may require little or no attentional resources (Cohen, Ivry, \& Keele, 1990; Curran \& Keele, 1993). It is therefore possible that mind-wandering may not impact implicit learning at all. There is also reason to suspect that mind-wandering might even improve implicit learning. For example, the competition between verbal and implicit systems theory (COVIS; Ashby, Paul, \& Maddox, 2011) was developed in an attempt to recognize that categorization is mediated by multiple learning systems. This theory suggests a competition between verbal and implicit systems. On the basis of COVIS, one might suspect that mind-wandering, by utilizing verbal processing that relies on the phonological loop (Teasdale et al., 1995), could release its interference with the implicit system. If so, this freeing of the implicit system could potentially enhance learning. In addition, recent work by Stillman, Feldman, Wambach, Howard, and Howard (2014) showed that trait mindfulness (i.e., the ability to maintain periods of sustained nondistraction) was negatively associated with implicit learning. This work suggested that mindlessness, or mind-wandering, may be positively related to implicit learning.

\section{Present study}

In order to investigate the role of task focus in implicit learning, in the present study we utilized a standard SRT task, alternating periods of the target sequence (i.e., a pattern) with random sequences. In order to measure the extent of attention directed toward the task, we embedded thought probes in the task that asked participants to indicate the degree to which their attention was focused on the task or on task-unrelated concerns. In addition, we collected scale measures of mind- 
wandering that included (a)the Imaginal Processes Inventory (IPI; Singer \& Antrobus, 1972), (b) the Attention-Related Cognitive Errors Scale (ARCES; Carriere, Cheyne, \& Smilek, 2008), and (c) the Memory Failures Scale (MFS; Cheyne, Carriere, \& Smilek, 2006).

Uncovering the relationship between mind-wandering and implicit learning would help establish the role of attention in implicit sequence learning. If implicit learning operates automatically, without requiring attentional resources, there should be no significant relationship between mind-wandering and implicit learning. If attention is necessary and/or helpful for implicit learning, a negative relationship between mindwandering and implicit learning might emerge. If, however, task focus impairs implicit learning, then we would expect a positive relationship between mind-wandering and implicit learning: Mind-wandering would actually aid implicit learning.

\section{Method}

\section{Participants}

In all, 74 participants were recruited for the study (48 female, 26 male; mean age $=18.85$ years, $S D=1.48)$. This study was granted ethical approval by the University of California, Santa Barbara, Ethics Committee, and written informed consent was acquired from every participant prior to participation.

\section{SRT task}

Four boxes were presented in a horizontal row at the center of the screen. An asterisk appeared in one of the four locations (referred to as 1-4, from left to right) for $1,000 \mathrm{~ms}$. Participants were given instructions to respond using their right hand in response to the location of the asterisk, as quickly and accurately as possible, using the $\mathrm{H}, \mathrm{J}, \mathrm{K}$, and L keys on the keyboard. The keys were in a "direct" spatial mapping to the target locations (such that an asterisk appearing in Location 1 would require an " $\mathrm{H}$ " keypress with the index finger, an asterisk appearing in Location 2 would require a "J" keypress with the middle finger, etc.). The target sequence consisted of 12 locations (1-2-1-4-2-3-4-1-3-2-4-3) and was created so that each location occurred three times and each possible transition (e.g., 1-2, 1-3, etc.) was never repeated (see Shanks \& Channon, 2002). The random sequences also consisted of 12 locations that were created on the basis of the same parameters, but importantly, these sequences all differed from one another (and the target sequence). A total of 624 trials were presented. Each half of the experiment contained 312 individual trials (96 random, 216 target). Random sequences (R) were interspersed with the presentation of the target sequence (T), as follows: $\mathrm{R}(2) \mathrm{T}(6) \mathrm{R}(2) \mathrm{T}(6) \mathrm{R}(2) \mathrm{T}(6) \mathrm{R}(2) \ldots$
Twelve thought probes were administered pseudorandomly (six during random sequences, six during target sequences). At the probes, participants were asked: "In the moments prior to the probe, was your attention focused: (1)Completely on the task, (2)Mostly on the task, (3)On both the task and unrelated concerns, (4)Mostly on unrelated concerns, (5) Completely on unrelated concerns?"

Although we recognize that more target trials were presented than random trials, we opted to choose parameters that have been used and are known to be conducive to implicit learning (i.e., enough target trials to facilitate learning). Given the spaced-out, pseudorandom nature of the design - that every 12 trials there was the potential for a thought probe-we did not expect this imbalance (especially given the low explicit awareness of target trials) to differentially influence thought probe responses and/or implicit learning. Also, we did not expect that interrupting random sequences with probes more often than target sequences could influence the main outcome, which was based on individual differences in mind-wandering and implicit learning.

\section{Explicit memory test}

At the conclusion of the experiment, participants were queried about the presence of a repeating pattern of target locations in the task. Specifically, they were told, "You may not have noticed, but there was a pattern embedded within some of the trials. Please use the 5-point scale below to rate how confident you are that you discovered the sequence." Next, they were asked to try to reproduce the pattern, which they were told could be up to 15 items, using the same response keys and mapping as in the main experiment. Accuracy was measured as the probability of generating the correct key response in the correct place in the sequence, chance $=.25$.

\section{Scales}

Finally, participants were administered the following scales to measure mind-wandering in everyday life.

Imaginal processes inventory Participants responded to Part I of the IPI, a 24-item questionnaire used to assess the frequency of daydreams (as an index of mind-wandering) and night dreaming (Singer \& Antrobus, 1972). Each question has five alternatives, with responses ranging from infrequent to frequent. A mean was calculated for both daydreaming and night dreaming across items, where a higher mean score indicated that the participant experienced a greater number of daydreams/night dreams.

Attention-related cognitive errors scale The ARCES (Carriere, Cheyne, \& Smilek, 2008) measures the frequency of everyday cognitive failures that are most likely caused by a 
lapse of attention. Participants use a scale of five possible responses, ranging from 1 (never) to 5 (very often). The mean across items was calculated, and a higher mean score indicated more attention-related cognitive errors.

Memory failures scale Participants completed the 12-item MFS (Cheyne, Carriere, \& Smilek, 2006) in order to assess everyday memory failures that are minimally explained by attentional errors. The scale includes items such as "I forget what I went to the supermarket to buy," to which participants respond on a 5-point scale ranging from 1 (never) to 5 (very often). The mean score was calculated, with a high score indicating that a participant was more prone to everyday memory failures.

\section{Results}

Of the 74 participants, four were excluded from the analysis for an accuracy $2.5 S D$ s below the mean $(<46 \%)$. The percent-correct SRT performance for the remaining participants was high $(M=92.5, S D=11.09)$. The mean RT for the target sequence was $419.50 \mathrm{~ms}(S D=60.95)$, and the RT for the random sequence was $435.95 \mathrm{~ms}(S D=58.29)$. A oneway repeated measures analysis of variance (ANOVA) revealed a significant effect of sequence type (target/random), $F(1,69)=43.16, p<.0001$. Mean confidence in learning the sequence was low $(M=2.18, S D=1.15)$, and accuracy at recreating items from the target sequence was no better than chance (accuracy $=21.43 \%, S D=13.94$ ), suggesting that explicit knowledge was not responsible for the effect of the faster RTs to target sequences. Moreover, the effect of the faster RTs to target sequences was significant even for those individuals $(N=24)$ who were least confident in identifying the target sequence (those who chose 1, Not confident at all, from the 5-point scale) $[F(1,22)=15.30, p<.001]$.

The mean response to thought probes across participants was $2.91(S D=0.80)$, corresponding closely to the middle scale item, reporting that attention was On both the task and unrelated concerns. We observed no difference between the random $(M=2.94, S D=0.83)$ and target thought probe responses $(M=2.93, S D=0.84)[F(1,69)=0.08, p=.76]$. When we assessed the relationship between task focus and implicit learning, there was a significant negative correlation between an individual's difference-score indices of implicit learning (random RT - target RT) and the individual's mean degree of mind-wandering (Fig. $1 ; r=-.31, p=.009$ ). Diminished task focus was associated with a smaller implicit-learning effect. This relationship was further investigated using a median split to create high- and low-mindwandering groups (see Fig. 2). A mixed-model ANOVA with Mind-Wandering Group (high/low) and Condition (random/ target) as factors revealed no main effect of mind-wandering on RTs $[F(1,68)=0.59, p=.44]$, a significant effect of condition $[F(1,68)=55.77, p<.0001]$, and a significant MindWandering Group $\times$ Condition interaction $[F(1,68)=9.63, p$ $=.003]$. This analysis revealed that the high-mind-wandering group was particularly slow on the target sequence. Additionally, the scale measures of mind-wandering in daily life (DDQ, MFS, and ARCES) were each negatively associated with implicit learning, although these relationships were not statistically significant $(r \mathrm{~s}=-.15,-.12$, and -.08 , respectively; all $p \mathrm{~s}>.20$ ).

\section{Discussion}

In the present study, we used a novel approach to investigate the effect of attention on implicit sequence learning. Previous studies had addressed this question by using a distractor task and comparing single-task (implicit) versus dual-task (implicit + distractor) conditions. Although this has been the dominant approach when addressing the question, authors have pointed out important limitations of this approach (Cohen et al., 1990; Curran \& Keele, 1993; Frensch et al., 1998; Hsiao \& Reber, 1998; Schvaneveldt \& Gomez, 1998; Shanks \& Channon, 2002; Stadler, 1995). In particular, the major theoretical limitation is that, with this procedure, one cannot fully rule out that the distractor task does more than just distract attention away from the task or that the distractor task interferes with the learning processes or how the SRT task is executed (see Shanks, 2003, for an extensive discussion of these issues). The aim of the present study was to offer a novel approach to address this question.

The results showed evidence of implicit learning, replicating earlier studies. That is, an RT advantage emerged for patterned as compared to random sequences, while participants reported low confidence and chance-level accuracy at reproducing the learned sequences. Second, and more importantly, we found that mind-wandering was associated with a diminished RT advantage for patterned versus random sequences, a finding that suggests that focusing attention on the task facilitates implicit sequence learning. This result is consistent with recent findings using the typical distractor task approach (Shanks \& Channon, 2002; Shanks et al., 2005). Importantly, the finding is not due to a general interference by thought probes, since all participants received thought probes, and learning was associated only with specific responses to these probes. Although the results from the questionnaire data were less clear, they were in the same direction, and it is plausible that weaker results would emerge for such "trait" measures than for the acute mind-wandering episodes assessed during the task.

It admittedly remains difficult to provide definitive prove that all of the learning that occurred in the SRT task was truly implicit. Although participants' accuracy did not differ from 


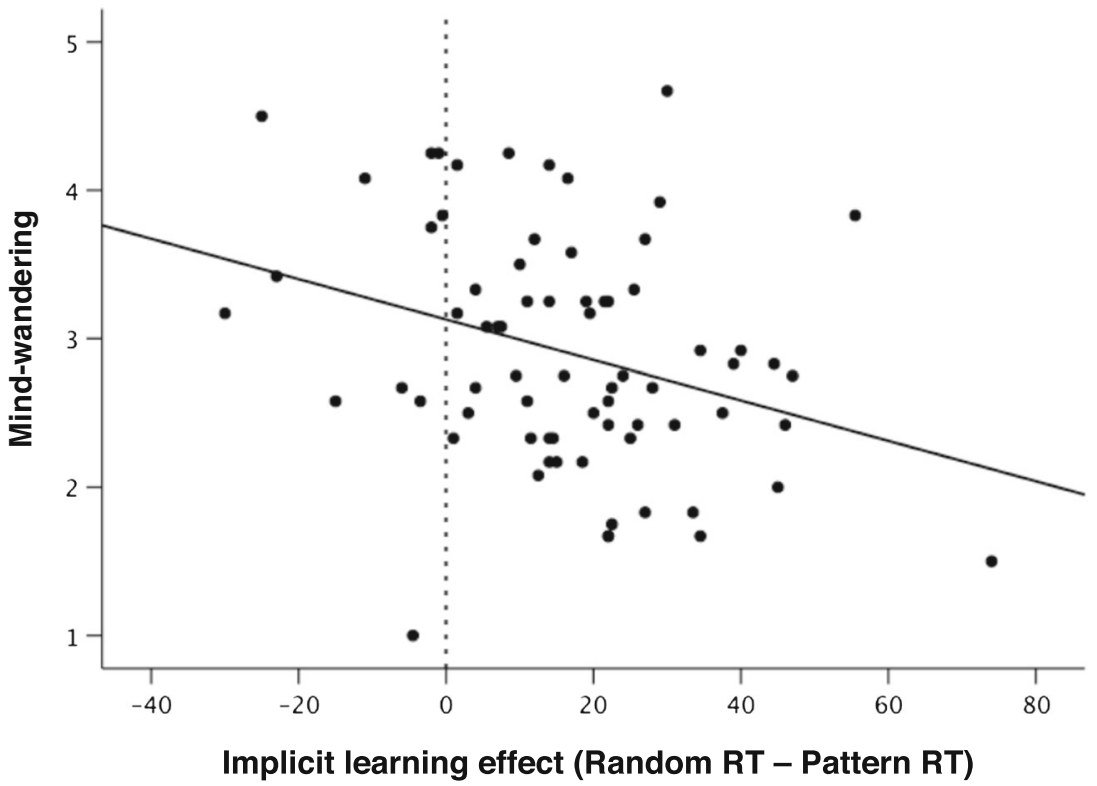

Fig. 1 Correlations between implicit learning and mean thought probe scores

chance (and was in fact numerically below chance level) when they were asked to reproduce the target sequence, and although participants reported low confidence in having detected a repeating sequence, their confidence ratings were not at the scale minimum. Thus, it remains possible that participants learned some components of the sequence explicitly. This would raise the possibility that mind-wandering might have interfered only with the part that had been learned explicitly, and not necessarily with implicit learning. Although we cannot fully exclude this possibility, we deem it unlikely that this effect would be sufficiently strong to drive the correlation between the RT advantage for the target sequence and mindwandering. However, further research will be needed to more closely examine the possibility.

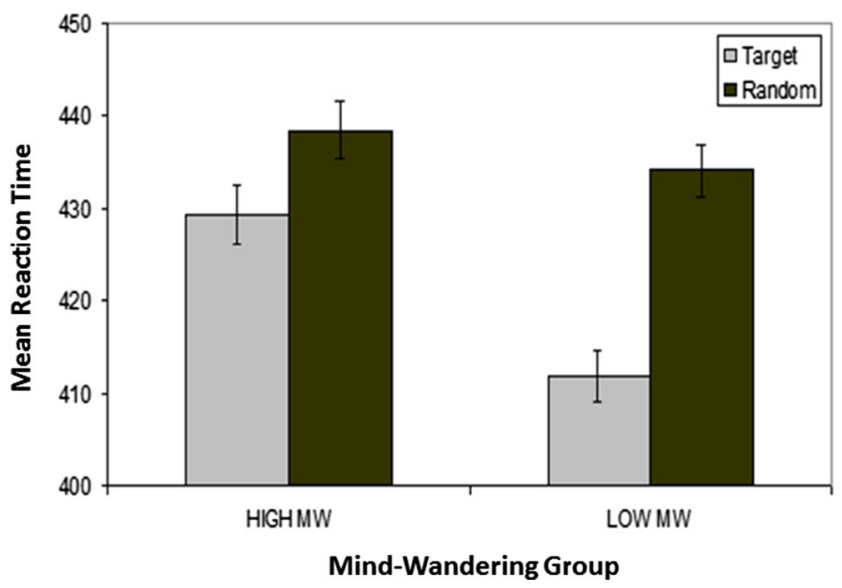

Fig. 2 Reaction times for target versus random sequences, based on a median split of the mind-wandering (MW) score. Error bars representing $95 \%$ confidence intervals for the within-subjects effect are plotted for this figure using methods taken from Loftus and Masson (1994)
Why would mind-wandering interfere with implicit sequence learning? On the basis of different literatures, one could predict negative effects, no effects, or even positive effects. So, in light of the present findings, it is important to understand the mechanism through which mind-wandering, at least under the present conditions (in the SRT task as it was used here), impairs learning. The mind-wandering state entails at least two processes: a decoupling of attention from perceptual input that affords an internal train of thought, and episodic memory processes that contribute the mental contents that are at odds with the task in hand (Smallwood, 2013). Plausibly, the intermittent decoupling of attention from perception that occurs during mind-wandering could reduce a participant's capacity to build up an implicit model of the patterns. Alternatively, it could be that the episodic memory processes that occur during mind-wandering also play a role in implicit learning, leading to the decrement that we found for individuals with frequent mind-wandering episodes. Given that the decrement was specific to the patterned trials, it is possible that, as in other distractor tasks, task-unrelated thoughts "fill up" the interval between task stimuli, thus reducing the number of successive stimuli that can be maintained in working memory. These results are consistent with recent work by Thomson, Smilek, and Besner (2014), revealing greater effects on mind-wandering with deep than with shallow encoding. Presumably, the acquisition of these hidden complex sequences requires deep encoding, and as such, is impacted by mind-wandering. Perhaps future work can distinguish different off-task states. For example, a distinction between "tuning out" versus "zoning out" (Smallwood, McSpadden, \& Schooler, 2007) could further refine our understanding of how mind-wandering impacts implicit 
learning; it is plausible that "zoning out" would be more detrimental than "tuning out."

Is it still possible that mind-wandering could, under some conditions, improve implicit learning? There are findings consistent with this idea, including a recent study showing that trait mindfulness (i.e., the ability to maintain periods of sustained nondistraction) was negatively associated with implicit learning (Stillman et al., 2014). Likewise, it is possible that a different type of implicit learning task-for example, one with less of a response component-might have different attentional demands. However, further work will be needed to test these ideas.

The present results provide strong evidence for the negative relationship between mind-wandering and implicit learning. At least in the context of an SRT task, implicit learning benefits from attention. This study has also addressed an important methodological issue associated with earlier work, through the use of experience sampling. Other studies investigating the role of attention in implicit learning could benefit from the experience-sampling probe approach introduced in the present article.

Author note M.S.F., J.M.B., C.M.Z., J.S., and J.W.S. are supported through United States Department of Education Grant Number R305A110277, awarded to J.S. and J.W.S. The content of this article does not necessarily reflect the position or policy of the US Government, and no official endorsement should be inferred.

\section{References}

Ashby, F. G., Paul, E. J., \& Maddox, W. T. (2011). COVIS. In E. M. Pothos \& A. J. Wills (Eds.), Formal approaches in categorization (pp. 65-87). Cambridge: Cambridge University Press.

Carriere, J. S. A., Cheyne, J. A., \& Smilek, D. (2008). Everyday attention lapses and memory failures: The affective consequences of mindlessness. Consciousness and Cognition, 17, 835-847. doi:10.1016/j. concog.2007.04.008

Cheyne, J. A., Carriere, J. S. A., \& Smilek, D. (2006). Absent-mindedness: Lapses of conscious awareness and everyday cognitive failures. Consciousness and Cognition, 15, 578-592. doi:10.1016/j. concog.2005.11.009

Cleeremans, A., \& Jiménez, L. (1998). Implicit sequence learning: The truth is in the details. In M. A. Stadler \& P. A. Frensch (Eds.), Handbook of implicit learning (pp. 323-364). Thousand Oaks: Sage.

Cohen, A., Ivry, R. I., \& Keele, S. W. (1990). Attention and structure in sequence learning. Journal of Experimental Psychology: Learning, Memory, and Cognition, 16, 17-30. doi:10.1037/0278-7393.16.1. 17

Curran, T., \& Keele, S. W. (1993). Attentional and nonattentional forms of sequence learning. Journal of Experimental Psychology: Learning, Memory, and Cognition, 19, 189-202. doi:10.1037/ 0278-7393.19.1.189

Filoteo, J. V., Lauritzen, S., \& Maddox, W. T. (2010). Removing the frontal lobes The effects of engaging executive functions on perceptual category learning. Psychological Science, 21, 415-423. doi:10. 1177/0956797610362646
Franklin, M. S., Smallwood, J., \& Schooler, J. W. (2011). Catching the mind in flight: Using behavioral indices to detect mindless reading in real time. Psychonomic Bulletin \& Review, 18, 992-997. doi:10. 3758/s13423-011-0109-6

Frensch, P. A., Lin, J., \& Buchner, A. (1998). Learning versus behavioral expression of the learned: The effects of a secondary tone-counting task on implicit learning in the serial reaction task. Psychological Research, 61, 83-98. doi:10.1007/s004260050015

Hsiao, A. T., \& Reber, A. S. (1998). The role of attention in implicit sequence learning: Exploring the limits of the cognitive un- conscious. In M. A. Stadler \& P. A. Frensch (Eds.), Handbook of implicit learning (pp. 471-494). Thousand Oaks: Sage.

Kane, M. J., Brown, L. H., McVay, J. C., Silvia, P. J., Myin-Germeys, I., $\&$ Kwapil, T. R. (2007). For whom the mind wanders, and when: An experience-sampling study of working memory and executive control in daily life. Psychological Science, 18, 614-621. doi:10.1111/j. 1467-9280.2007.01948.x

Keele, S. W., Ivry, R., Mayr, U., Hazeltine, E., \& Heuer, H. (2003). The cognitive and neural architecture of sequence representation. Psychological Review, 110, 316-339. doi:10.1037/0033-295X.110. 2.316

Killingsworth, M. A., \& Gilbert, D. T. (2010). A wandering mind is an unhappy mind. Science, 330, 932. doi:10.1126/science.1192439

Loftus, G. R., \& Masson, M. E. J. (1994). Using confidence intervals in within-subject designs. Psychonomic Bulletin \& Review, 1, 476 490. doi:10.3758/BF03210951

Mooneyham, B. W., \& Schooler, J. W. (2013). The costs and benefits of mind-wandering: A review. Canadian Journal of Experimental Psychology, 67, 11-18. doi:10.1037/a0031569

Mrazek, M. D., Smallwood, J., Franklin, M. S., Chin, J. M., Baird, B., \& Schooler, J. W. (2012). The role of mind-wandering in measurements of general aptitude. Journal of Experimental Psychology: General, 141, 788-798. doi:10.1037/a0027968

Nemeth, D., Janacsek, K., Polner, B., \& Kovacs, Z. A. (2013). Boosting human learning by hypnosis. Cerebral Cortex, 23, 801-805. doi:10. 1093/cercor/bhs068

Newell, B., Moore, C. P., Willis, A. J., \& Milton, F. (2013). Reinstating the frontal lobes? Having more time to think improves implicit perceptual categorization: A comment on Filoteo, Lauritzen, and Maddox (2010). Psychological Science, 24, 386-389. doi:10.1177/ 0956797612457387

Nissen, M. J., \& Bullemer, P. (1987). Attentional requirements of learning: Evidence from performance measures. Cognitive Psychology, 19. 1-32. doi:10.1016/0010-0285(87)90002-8

Schmidtke, V., \& Heuer, H. (1997). Task integration as a factor in secondary-task effects on sequence learning. Psychological Research, 60, 53-71. doi:10.1007/BF00419680

Schooler, J. W., Reichle, E. D., \& Halpern, D. V. (2004). Zoning out while reading: Evidence for dissociations between experience and metaconsciousness. In D. T. Levin (Ed.), Thinking and seeing: Visual metacognition in adults and children (pp. 203-226). Cambridge: MIT Press.

Schvaneveldt, R. W., \& Gomez, R. L. (1998). Attention and probabilistic sequence learning. Psychological Research, 61, 175-190. doi:10. $1007 / \mathrm{s} 004260050023$

Seger, C. A. (1994). Implicit learning. Psychological Bulletin, 115, 163 196. doi:10.1037/0033-2909.115.2.163

Seli, P., Carriere, J. S. A., Thomson, D. R., Cheyne, J. A., Martens, K. A. E., \& Smilek, D. (2014). Restless mind, restless body. Journal of Experimental Psychology: Learning, Memory, and Cognition, 40, 660-668. doi:10.1037/a0035260

Shanks, D. R. (2003). Attention and awareness in "implicit" sequence learning. In L. Jimenez (Ed.), Attention and implicit learning (pp. 11-42). Amsterdam: Benjamins.

Shanks, D. R., \& Channon, S. (2002). Effects of a secondary task on "implicit" sequence learning: Learning or performance? 
Psychological Research, 66, 99-109. doi:10.1007/s00426-0010081-2

Shanks, D. R., Rowland, L. A., \& Ranger, M. S. (2005). Attentional load and implicit sequence learning. Psychological Research, 69, 369382. doi:10.1007/s00426-004-0211-8

Singer, J. L., \& Antrobus, J. S. (1972). Daydreaming, imaginal processes, and personality: A normative study. In P. W. Sheehan \& J. S. Antrobus (Eds.), The function and nature of imagery (pp. 175202). New York: Academic Press.

Smallwood, J. (2013). Distinguishing how from why the mind wanders: A process-occurrence framework for self-generated mental activity. Psychological Bulletin, 139(3), 519-535. doi:10.1037/a0030010

Smallwood, J., \& Andrews-Hanna, J. (2013). Not all minds that wander are lost: The importance of a balanced perspective on the mindwandering state. Frontiers in Psychology, 16, 441. doi:10.3389/ fpsyg.2013.00441

Smallwood, J., McSpadden, M., \& Schooler, J. W. (2007). The lights are on but no one's home: Meta-awareness and the decoupling of attention when the mind wanders. Psychonomic Bulletin \& Review, 14, 527-533. doi:10.3758/BF03194102

Smallwood, J., McSpadden, M., \& Schooler, J. W. (2008). When attention matters: The curious incident of the wandering mind. Memory \& Cognition, 36, 1144-1150. doi:10.3758/MC.36.6.1144

Smallwood, J., Obonsawin, M., \& Heim, D. (2003). Task unrelated thought: The role of distributed processing. Consciousness and Cognition, 12, 169-189. doi:10.1016/S1053-8100(02)00003-X
Stadler, M. A. (1995). Role of attention in implicit learning. Journal of Experimental Psychology: Learning, Memory, and Cognition, 21, 674-685. doi:10.1037/0278-7393.21.4.674

Stillman, C. M., Feldman, H., Wambach, C. G., Howard, J. H., \& Howard, D. V. (2014). Dispositional mindfulness is associated with reduced implicit learning. Consciousness and Cognition, 28, 141150. doi:10.1016/j.concog.2014.07.002

Teasdale, J. D., Dritschel, B. H., Taylor, M. J., Proctor, L., Lloyd, C. A., Nimmo-Smith, I., \& Baddeley, A. D. (1995). Stimulus-independent thought depends on central executive resources. Memory \& Cognition, 23, 551-559. doi:10.3758/BF03197257

Teasdale, J. D., Proctor, L., Lloyd, C. A., \& Baddeley, A. D. (1993). Working memory and stimulus-independent thought: Effects of memory load and presentation rate. European Journal of Cognitive Psychology, 5, 417-433. doi:10.1080/ 09541449308520128

Thomson, D. R., Besner, D., \& Smilek, D. (2013). In pursuit of off-task thought: Mind wandering-performance trade-offs while reading aloud and color naming. Frontiers in Psychology, 4, 360. doi:10. 3389/fpsyg.2013.00360

Thomson, D. R., Smilek, D., \& Besner, D. (2014). On the asymmetric effects of mind-wandering on levels of processing at encoding and retrieval. Psychonomic Bulletin \& Review, 21, 728-733. doi:10. 3758/s13423-013-0526-9 